\title{
A Simple Analysis of Texture Induced Friction Reduction Based on Surface Roughness Ratio
}

\author{
Jiaxin Ye \\ Hefei University of Technology \\ Jiazhou Xuan \\ Hefei University of Technology \\ Yongliang Qiao \\ Shanghai Aerospace Control Technology Institute \\ Yifan Zhang \\ Hefei University of Technology \\ Haiyang Zhang \\ Hefei University of Technology \\ Jimin Xu \\ Hefei University of Technology \\ Xiaojun Liu \\ Hefei University of Technology \\ Kun Liu ( $\square$ liukun@hfut.edu.cn ) \\ Hefei University of Technology https://orcid.org/0000-0002-7502-7510
}

\section{Tribology Methods}

Keywords: texture, friction reduction, surface roughness ratio

Posted Date: February 11th, 2021

DOI: https://doi.org/10.21203/rs.3.rs-182577/v1

License: (c) (i) This work is licensed under a Creative Commons Attribution 4.0 International License.

Read Full License 


\title{
A Simple Analysis of Texture Induced Friction Reduction Based on Surface Roughness Ratio
}

\author{
Jiaxin Ye ${ }^{1}$, Jiazhou Xuan ${ }^{1}$, Yongliang Qiao ${ }^{2}$, Yifan Zhang ${ }^{1}$, Haiyang Zhang ${ }^{1}$, Jimin $\mathrm{Xu}^{1}$, Xiaojun Liu ${ }^{1}$, \\ Kun Liu ${ }^{1, *}$ \\ ${ }^{1}$ Institute of Tribology \\ Hefei University of Technology \\ Hefei, Anhui, \\ China \\ ${ }^{2}$ Shanghai Aerospace Control Technology Institute \\ Shanghai, China
}

\begin{abstract}
The effect of surface texture on friction reduction under fluid lubrication has been broadly acknowledged in the tribology community. However, the lack of understanding of the underlying mechanisms remains a challenge for the advancement of textured enhanced lubrication. Numerous models have been proposed, but they are almost all based on the hydrodynamic effect alone and have proven cumbersome, system specific and unreflective of the beneficial secondary lubrication provided by the residual lubricants within the texture. This paper presents a simple analysis of texture induced friction reduction based on the actual liquid-solid interface area and the secondary lubrication hypothesis. A simple model based on the surface roughness ratio (the ratio between the actual and projected solid surface area) of the textured surface was proposed which 1) is simple, intuitive, quantitative and sensitive to texture shape and area fraction; 2) directly reflects proposed secondary lubrication mechanisms; 3) reflects the general data trend in the collected literature. By focusing on the variations of key texture parameters, the proposed model combined with a sampling of independent studies in literature has demonstrated that 1$)$ the effect of increased pit depth-to-diameter ratio $(d / D)$ on friction reduction is most significant between 0.01 and $0.2 ; 2$ ) further increase in $d / D$ only marginally affects the friction coefficient; 3) texture's area fraction plays a much weaker role than the depth/diameter ratio in friction reduction. By quantitatively isolating the secondary lubrication effects, this model may help to link disparate studies in the literature while providing defensible quantitative insights into texture induced lubrication mechanisms.
\end{abstract}

keywords: texture; friction reduction; surface roughness ratio

\section{*corresponding author}

Kun Liu, Ph.D.

Institute of Tribology

Hefei University of Technology

Hefei, Anhui, China, 230051

liukun@hfut.edu.cn

+86-551-62901756-2729 


\subsection{Introduction}

Tremendous effects have been made in literature in designing optimum laser-induced surface texturing (LST) to improve tribological properties of mechanical components under fluid lubricated conditions [1-5]. Texture design tools adopted include experimental [6-13], computational [1, 4, 14-23], analytical [3, 5, 24-29]. Lubrication conditions investigated include boundary [24, 30-32], mixed [11, 27, 33-36], EHL [14, 37-39] and hydrodynamic [6, 40-52]. Lubrication hypotheses include hydrodynamic [6, 7, 11, 33, 40, 41, 49-60], microrepository for lubricants $[41,49,50,55,58,61-65]$ or a combination of both $[41,49$, $50,55,58]$. Key design parameters include texture depth, density, shape, depth/diameter ratio $[44,66]$ etc.

The most common and extensively studied LST texture is orthogonal array of micro-pits with $1-50 \mu \mathrm{m}$ depth $(d)$ and up to a few hundreds of micrometers diameter $(D)$ which could reduce friction coefficient up to $50 \%$ compared with untextured surface $[3,5,11,67,68]$. Ramesha et al. studied the lubricating effect of surface micro-pits of much deeper depth and found a $90 \%$ friction reduction with micro-pits of $100 \mu \mathrm{m}$ depth [33]. To the authors' best knowledge, this is about the highest friction reduction reported in tribological surface texture literature.

Despite a large volume of literature on texture induced friction reduction, most applications rely on a case by case approach in texture optimization and there is no universal principle for LST design. Theoretical analysis solely based on the hydrodynamic effect have often proven cumbersome, system specific and could differ significantly from the experimental results when a single testing parameter (load, velocity, etc.) was changed [55]. Secondary lubrication mechanism provided by the residual lubricants within the texture are widely acknowledged, rarely quantified and as important as, if not more than, the hydrodynamic effect to the lubrication $[49,58,65,69,70]$.

It is well documented that metallic surface textures have some degree of friction or wear reduction under almost all lubrication conditions (starved, boundary, mixed, hydrodynamic, etc.) suggesting some common mechanisms at work. Based on this hypothesis and a survey of literature, we present here a simple analysis of LST induced friction reduction based on the secondary lubrication hypothesis and the surface roughness ratio of the textured surface. A simple model is proposed to corelate the surface roughness ratio with normalized friction coefficient and validated using data collected from the literature. 


\subsection{A Friction Reduction Model Based on Surface Roughness Ratio}

A common finding in almost every LST study in literature is a possible relation between friction reduction and a secondary lubrication mechanism provided by residual lubricants within the textured pits (often referred as microrepository for lubricants, lubricant reservoir, etc.) $[41,49,50,55,58,62-65]$. Although LST induced secondary lubrication can significantly impact friction reduction, it is not itself a quantifiable property; qualitative and subjective assessments remain as the 'gold standard' for describing this effect.

Wenzel model of contact angle:
roughness ratio: $r=\frac{A^{\prime}}{A} \quad \begin{gathered}\theta^{*}<\theta<90^{\circ} \\ \cos \theta^{*}=r \cos \theta\end{gathered}$
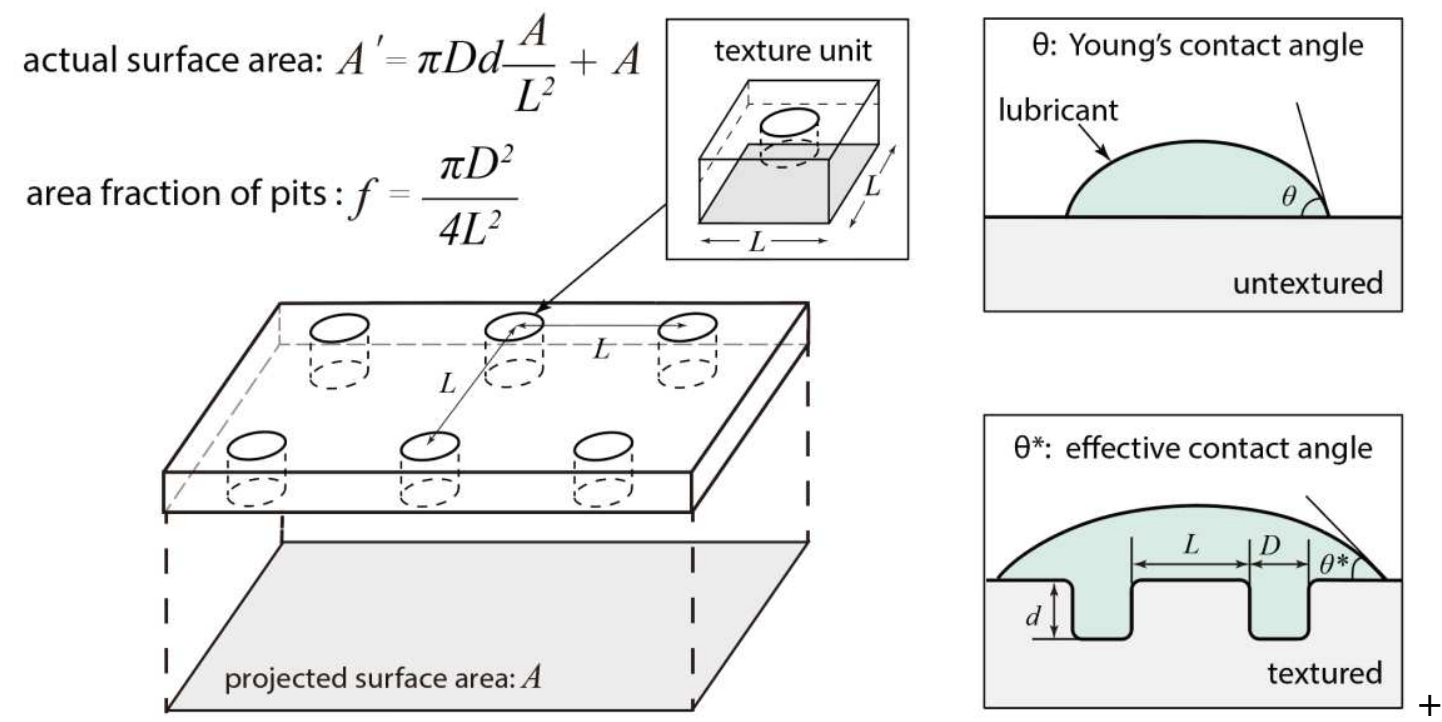

Fig. 1. A simple illustration of wettability of fluid lubricants on textured surfaces based on the classical Wenzel model [71]. The roughness ratio $(r)$ is defined as the quotient of the actual solid surface area $\left(A^{\prime}\right)$ and the projected surface area $(A)$. Surface texture generally introduces extra area of the lubricant drop that is in contact with the solid, which causes a decrease in the wetting angle for cases where the wetting angle is less than 90 degree.

One possible way in which LST could affect tribological properties is to change wettability of fluid lubricants on the textured surfaces $[64,72,73]$. In surface science community, surface wettability is often quantified using the contact angle between a small droplet of the target liquid and the solid surface. For rough or patterned surfaces (i.e. LST), minimization of the free energy leads to an equilibrium effective contact angle $\theta^{*}$, that accounts for the extra area of the drop that is in contact with the solid. The classical Wenzel model [71] suggests the 
effective wetting angle $\left(\theta^{*}\right)$ is a function of the surface roughness ratio $(r)$ and the ideal contact angle $(\theta)$ defined by the Young's equation and the relation is best described as:

$$
\cos \theta^{*}=r \cos \theta
$$

Eq. 1.

Here, the surface roughness ratio $(r)$ is defined as the ratio between the actual and projected solid surface area ( $r=1$ for an ideal smooth surface and $r>1$ for a rough one).

Fig. 1 best illustrates wettability of fluid lubricants on textured surfaces based on the classical Wenzel model. As most tribological surfaces have high wettability towards lubricants $(\theta<$ $\left.90^{\circ}\right)$, the introduction of texture increases the surface roughness ratio and decreases the wetting angle $\left(\theta^{*}<\theta\right)$. Orthogonal array of micro-pits is the most common and extensively studied texture in literature and is often defined using three parameters: the pit diameter $(D)$, depth $(d)$ and area fraction $(f)$ as shown in Fig.1. Extra surface area per a single repetitive texture unit as illustrated in Fig. 1 could be calculated as $\pi D d / L^{2}$. Here, $L$ is the center-tocenter distance of neighboring pits. The actual surface area for the whole surface, $A$ ', is the sum of the extra side area of the pits and the projected surface area, $A$ :

$$
A^{\prime}=\pi D d \frac{A}{L^{2}}+A
$$

The roughness ratio, $r$, is given by

$$
r=\frac{A^{\prime}}{A}=1+\frac{\pi D d}{L^{2}}
$$

As the area fraction of pits, $f$, could be written as

$$
f=\frac{\pi D^{2}}{4 L^{2}}
$$

Eq. 3 could be rewritten as

$$
r=\frac{A^{\prime}}{A}=1+\frac{\pi D d}{L^{2}}=1+4 f \frac{d}{D}
$$

In its definition, roughness ratio reflects the relative increase of counterface's real fluid-solid interface area from a flat surface when wetted with lubricants. There are quantities of reports in tribology literature that the increased fluid-solid interface area in textured surfaces often lead to increased wettability towards the lubricants $[64,72,73]$. Based on these observations, the fluid-solid interface area may similarly affect the secondary lubrication mechanism as increased interfacial area tends to retain more lubricants during sliding from increased surface 
wettability. And it is reasonable to expect a possible relation between the roughness ratio and the friction reduction on textured surfaces.

To test the above hypothesis, we collected a sampling of independent measurements on micro-pits texture induced friction lubrication from 24 independent studies [6, 11, 33, 40-47, 49-51, 54-56, 58-60, 62-65]. Together, they covered a wide range of material, load, speed and lubrication conditions. Fig. 2 shows the normalized friction coefficient plotted against the roughness ratio. The normalized friction coefficient is defined as the ratio between friction coefficient on textured counterface and that on untextured counterface under identical sliding conditions within each study (load, speed, lubricants, etc.). Generally, the normalized friction coefficient decreases with increased roughness ratio which supports our hypothesis. The best performance data with the lowest friction reduction within each independent study were tabulated in Table 1 in the Appendix.

Because there is no theory to predict any particular relationship a priori, we fit the complete dataset of 100 data points in Fig. 2 with a power-law function:

$$
\frac{\mu}{\mu_{0}}=a \cdot\left(1+4 \frac{d}{D} f\right)^{-k}=a \cdot r^{-k}
$$

Because Eq. 6 has to satisfy $\left(r, u^{\prime} u_{0}\right)=(1,1)$ as the ideal smooth surface is set as the reference, the value of $a$ is ensured to be unity. Quadrature regression analysis was conducted, and Fig. 2 shows the best fit in the dashed line which has a $k$ value of 3.19. The grey region represents the best-fit \pm mean deviation of the data from the model. 


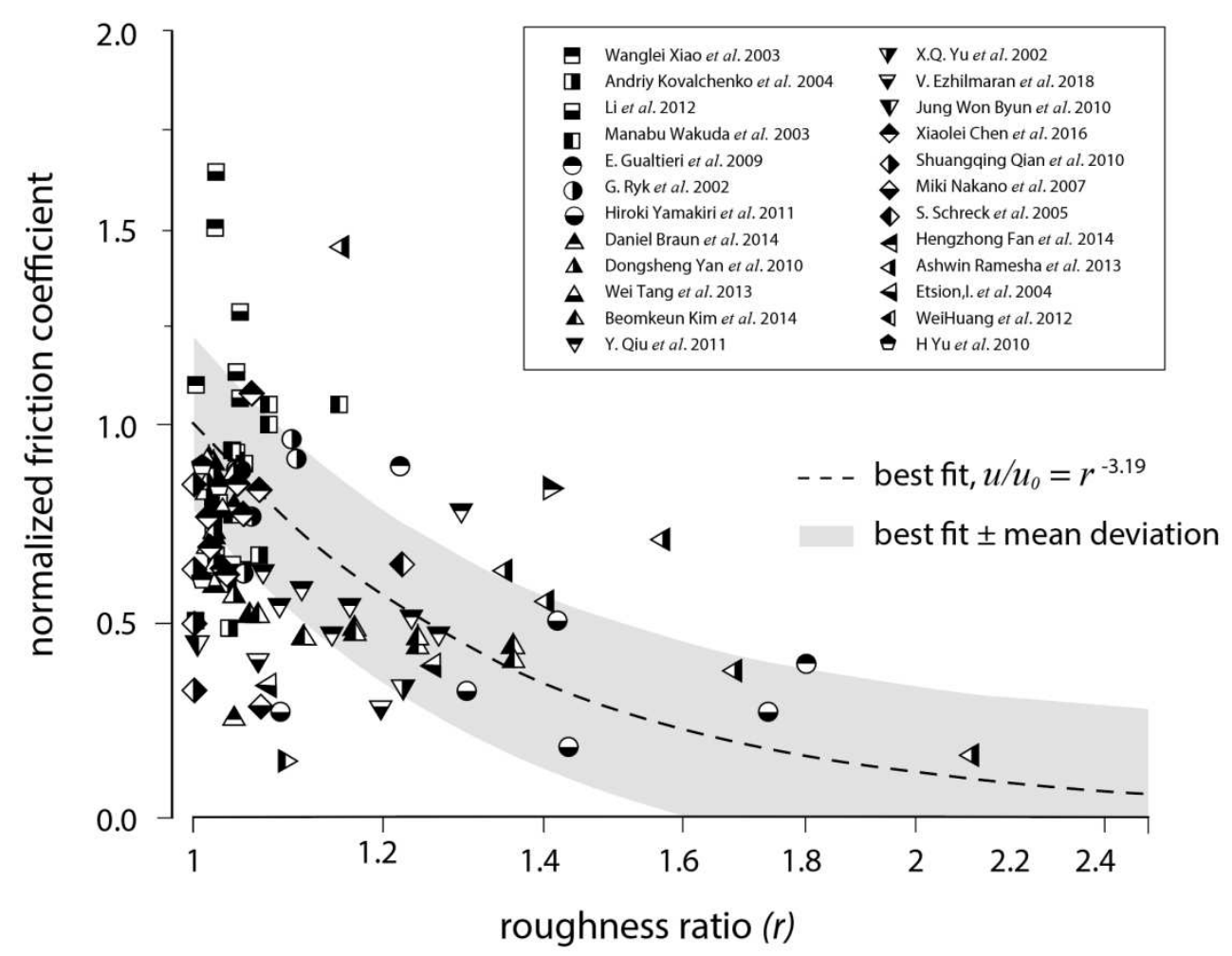

Fig. 2. Normalized friction coefficient plotted against counterface roughness ratio for a collection of literature results using orthogonal arrays of micro-pits texture under fluid lubrication. Normalized friction coefficient is defined as the ratio between friction coefficient on textured counterface and that on untextured counterface under identical sliding conditions (load, speed, etc.). Counterface roughness ratio is calculated using Eq. 5 and texture parameters provided in original studies. A power law function is used to fit the data and the best fit is shown in the dashed line. Grey area represents the mean deviation of the data from the model.

\subsection{Model Validation and Discussion}

A sensible model should not only reflect the physics of secondary lubrication, but also be able to predict the effects of key design parameters on friction reduction. In this section, we use the present model to analyze the effects of two parameters frequently adopted in literature on micro-pits texture design: pit's depth-to-diameter ratio and area fraction.

A large body of literature suggest micro-pits could act as small dynamic plain bearings under good lubrication and the pit depth-to-diameter ratio directly determines the hydrodynamic lubrication and the surface's load bearing ability [6, 7, 33, 41, 49, 50, 55, 56, 60, 61, 67]. This is further supported by the fact that friction reduction correlates more strongly with the pit depth-to-diameter ratio than with depth or diameter alone $[1,23,39,54,60,66]$. Pit depth-todiameter ratio with maximum friction reduction predicted using the hydrodynamic lubrication 
theory often lies between 0.01 and 0.2 [43-45, 55, 66, 68]. However, such optimums differ significantly between studies and not always coincide with the experimental results $[55,56]$. In the proposed model in Eq. 6, normalized friction coefficient is a strong function of the pit depth-to-diameter ratio and should decrease with increased $d / D$. To test the predictability of the model, a lower bound of normalized friction coefficient could be written as

$$
\left(\frac{\mu}{\mu_{0}}\right)_{\text {min }}=\left(1+4 \frac{d}{D}\right)^{-3.19}
$$

using the maximum area fraction value $(f=1)^{1}$. In theory, Eq. 7 represents the best possible case of friction reduction based on the roughness ratio hypothesis. Fig. 3 plots the normalized friction coefficient against the $d / D$ for the complete dataset in Fig. 2 and the lower bound was shown with the dashed line. The mean deviation of the data from the lower bound within the high depth/diameter ratio domain $(d / D>0.15)$ is 1.9 times of that within the low depth/diameter ratio domain $(d / D<0.15)$, and the two deviations were shown with two different shades of grey in Fig. 3. In summary, $91 \%$ of all data points were above the predicted lower bound and the date generally fits the trend predicted by Eq. 7, especially below 0.15 pit depth-to-diameter value. Fig. 5 in the Appendix plots the normalized friction coefficient against the $d / D$ for the best performance data within each independent study.

\footnotetext{
${ }^{1}$ In theory, $f_{\max }$ equals 0.785 for circular pits and 1 for rectangular pits. A $100 \%$ area fraction value was used here as a few studies cited here used rectangular shaped pits.
} 


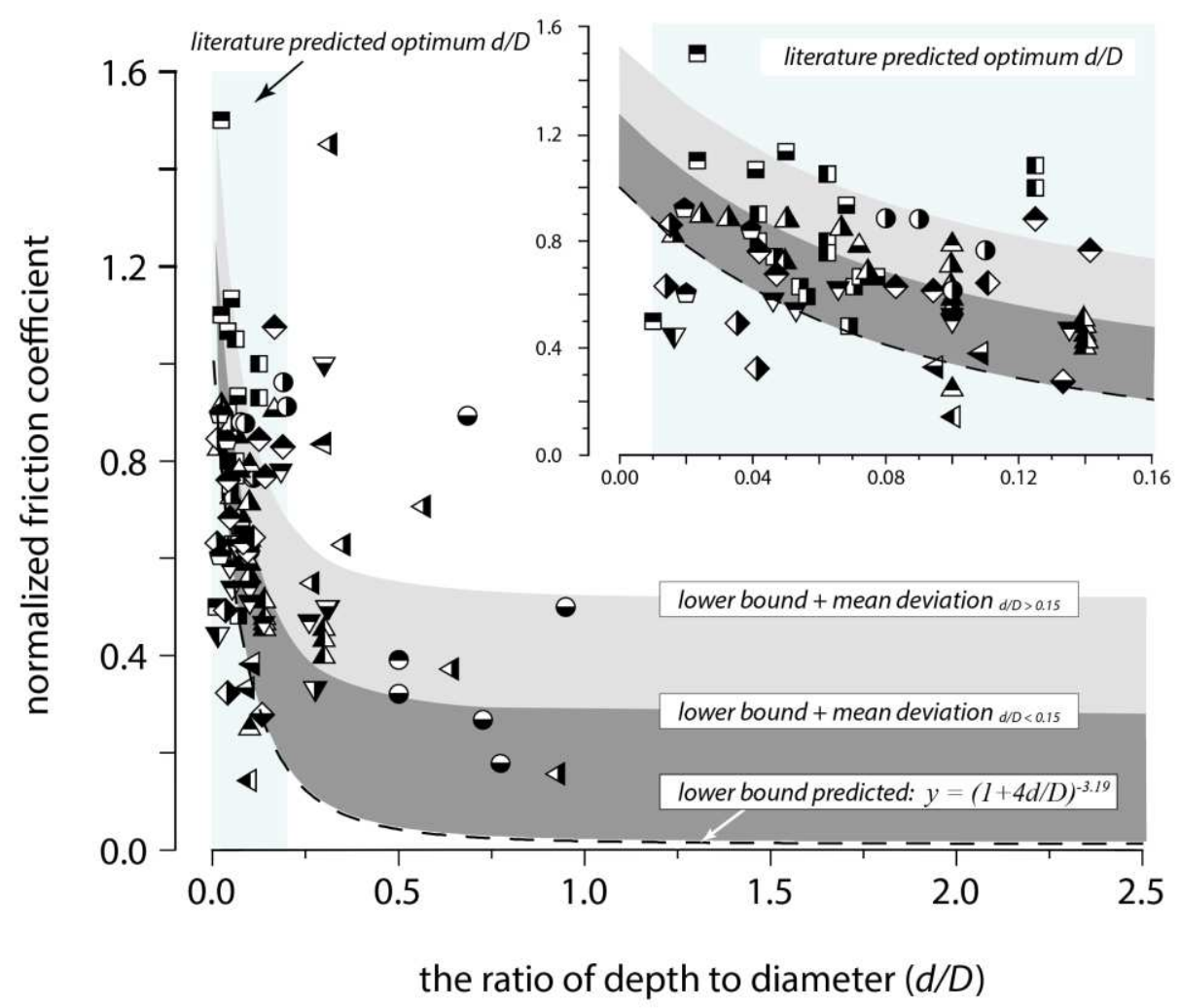

Fig. 3. Normalized friction coefficient plotted against the ratio between pit depth $(d)$ and diameter $(D)$ for the complete dataset in Fig. 2. The same legend as in Fig. 2 was used. The lower bound of friction reduction predicted by Eq. 7 was shown as the dashed line. Grey regions represent the mean deviation of the data from the lower bound within the high $(d / D>$ $0.15)$ and low $(d / D<0.15)$ depth/diameter ratio domains. $91 \%$ of data points were above the predicted lower bound which supports our model of friction reduction hypothesis.

Fig. 3 suggests the proposed model in this study reasonably reflects the general data trend in the collected literature: the normalized friction coefficient decreases rapidly when $d / D$ increases from 0 to 0.2 , and further increase in $d / D$ only marginally affects the friction coefficient. Interestingly, this critical domain of $d / D$ coincides with the optimum $d / D$ domain $(0.01,0.2)$ predicted in literature based on the hydrodynamic lubrication theory.

In Fig. 3, it is easier to understand the curve's tailing off at higher $d / D$ using the classical hydrodynamic or secondary lubrication theory as higher $d / D$ reduces the hydrodynamic effect and makes lubricant exchange across the pit edge more difficult. It is more difficult to consider the impact of higher $d / D$ on the lubricant film thickness from the surface wettability point of view. In physics, wetting of geometrically structured surfaces has been a focus of interest for decades. Studies on nanostructured surfaces have revealed that the initial fluid filling of a single pit does not depends on whether it stands alone or is part of an array [74]; whereas when the pits are close to saturation, the amount of fluids adsorbed depends strongly 
on the array as a whole and the detailed relations remain uncertain [75]. The key to solving such problem is to deepen our understanding of fluid adsorption and wetting transitions near individual wedges and cones [76] which is beyond the scope of this work.

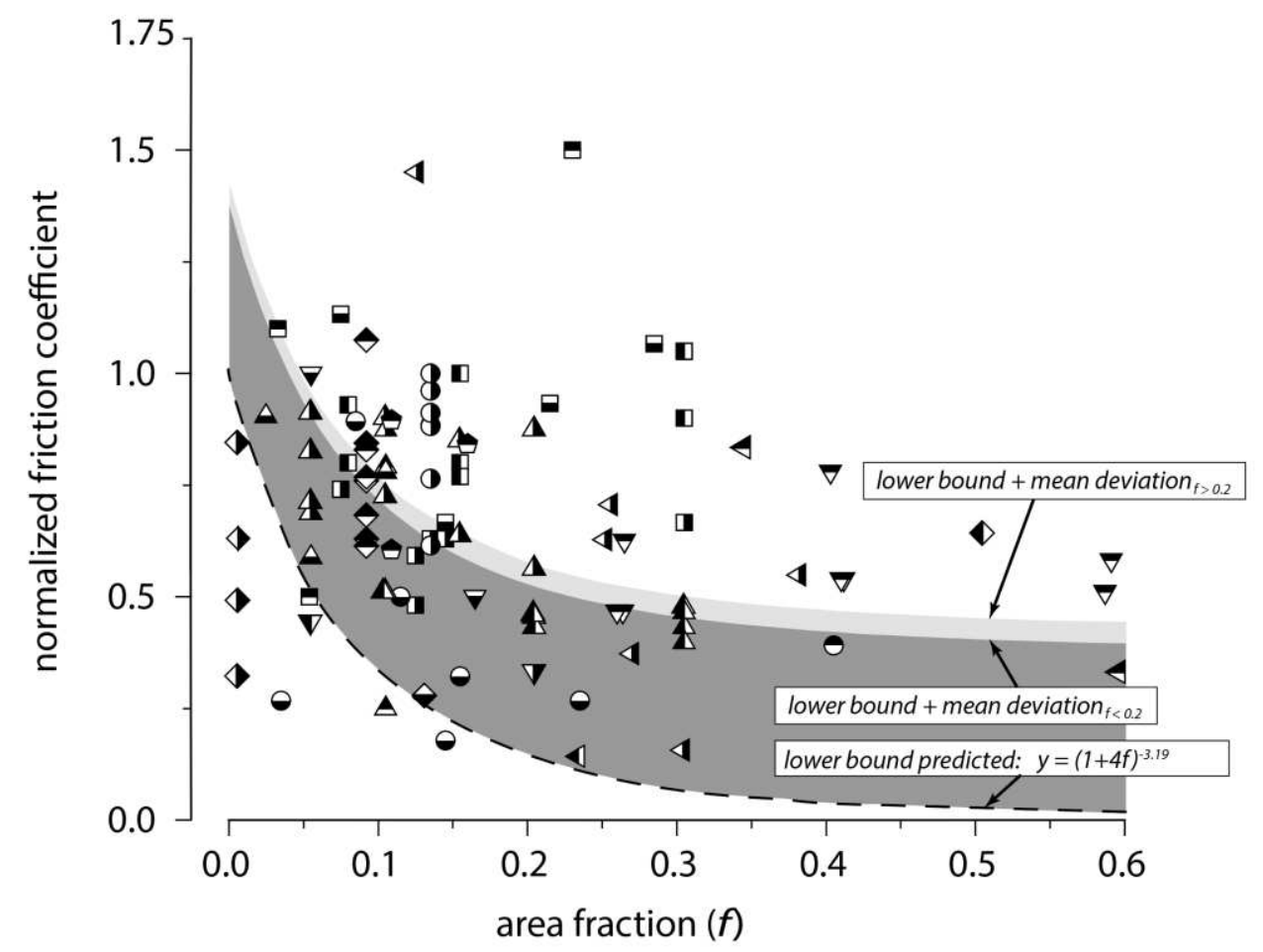

Fig. 4. Normalized friction coefficient plotted against the area fraction of pits $(f)$ for the complete dataset in Fig. 2. The same legend in Fig. 2 was used. The lower bound of friction reduction predicted by Eq. 8 was shown as the dashed line. Grey regions represent the mean deviation of the data from the lower bound within the high $(f>0.2)$ and low $(f<0.2)$ pit's area fraction domains. $91 \%$ of data points were above the predicted lower bound which supports our model of friction reduction hypothesis.

Another way to check the effectiveness of the model is to plot the normalized friction coefficient against the area fraction $f$ for the complete dataset in Fig. 2. The result is illustrated in Fig. 4 in which a lower bound was shown with the dashed line and could be written as

$$
\left(\frac{\mu}{\mu_{0}}\right)_{\min }=(1+4 f)^{-3.19}
$$

using the maximum $d / D$ value $\left(d / D_{\max } \sim 1\right)$ in the collected data. There are three interesting results here: first, $91 \%$ of all data points were above the predicted lower bound; second, mean deviation of the data from the lower bound is insensitive to area fraction $\left(\sigma_{f>0.2}=1.12 \sigma_{f<0.2}\right)$; third, no optimum area fraction was noticed in the overall dataset. In Fig. 4, the diminished 
correlation between the model and the data supports the literature hypothesis that textured pit's area fraction plays a much weaker role than the depth/diameter ratio in friction reduction $[1,60]$.

\subsection{Closing Remarks}

The tribology community broadly acknowledges the important effect of surface texture on friction reduction under fluid lubrication. However, the absence of a universal design principle has limited the adoption of LST in tribological design. To date, the community has limited insights for LST design directions and apparent contradictions in the literature have unclear sources that are likely related to uncharacterized differences in the secondary lubrication mechanism. Numerous friction reduction models have been proposed, but they were almost all based on the hydrodynamic effect alone and none have been widely adopted due largely to lack of portability, ease of use and sensitivity to the secondary lubrication. They are often developed to respond to a specific system and are of limited value for application to the more general field of literature. The roughness ratio model presented here is simple, intuitive, quantitative and sensitive to texture shape and area fraction. It was developed based on the secondary lubrication hypothesis and the surface wettability theory. A broad sampling of literature measurements showed the model reflected the general data trend in the collected literature. By focusing on the variations of key texture parameters, the proposed model has demonstrated that (1) the effect of increased $d / D$ on friction reduction is most significant between 0.01 and 0.2 depth-to-diameter ratio, (2) further increase in $d / D$ only marginally affects the friction coefficient, (3) pit's area fraction plays a much weaker role than the depth/diameter ratio in friction reduction. There is strong evidence to suggest that the optimum surface textures that are overwhelmingly observed in relevant studies are due to a coupled effect of hydrodynamic and secondary lubrication. The broad applicability of this method will prove useful for studying the effect of secondary lubrication on friction reduction. By understanding and isolating the effects of secondary lubrication, the method may also provide general quantitative insights into LST design which aims at friction reduction and lubrication. 


\section{$214 \quad 5.0$ Appendix}

215 Table 1. Table of the best performance data with the lowest friction reduction within each independent study in Fig. 2.

\begin{tabular}{|c|c|c|c|c|c|c|}
\hline $\begin{array}{c}\text { Refs } \\
\text { number }\end{array}$ & Authors & Lubricant & Pit Area Fraction, $f$ & $\begin{array}{l}\text { Pit Depth over Diameter, } \\
d / D\end{array}$ & $\begin{array}{l}\text { Roughness Ratio, } \\
r\end{array}$ & $\begin{array}{l}\text { Optimum Normalized } \\
\text { Friction Coefficient, } \\
\mu / \mu_{0}\end{array}$ \\
\hline 1 & X.Q. Yu et al. (2002) & Light oil & $20 \%$ & 0.28 & 1.22 & 0.33 \\
\hline 2 & G.Ryk et al. (2002) & SAE 40 & $13 \%$ & 0.1 & 1.05 & 0.62 \\
\hline 3 & Xiaolei Wang et al. (2003) & water & $4.9 \%$ & 0.01 & 1 & 0.5 \\
\hline 4 & Kovalchenko et al. (2004) & Mobil-10W30 & $12 \%$ & 0.07 & 1.03 & 0.48 \\
\hline 5 & E. Gualtieri et al. (2009) & Vanguards ST-46 & $40 \%$ & 0.5 & 1.8 & 0.39 \\
\hline 6 & Dongsheng Yan et al.(2010) & CD15W-40 engine oil & $10 \%$ & 0.1 & 1.04 & 0.56 \\
\hline 7 & Hiroki Yamakiri et al. (2011) & water & $14 \%$ & 0.77 & 1.43 & 0.18 \\
\hline 8 & Y. Qiu et al. (2011) & SAE 30 oil & $25 \%$ & 0.26 & 1.27 & 0.47 \\
\hline 9 & Li et al. (2012) & L-AN32 oil, $\quad \gamma=33.5 \mathrm{~mm}^{2} / \mathrm{s}$ & $14 \%$ & 0.07 & 1.04 & 0.64 \\
\hline 10 & Ashwin Ramesha et al (2013) & $85 \mathrm{~W}-140$ gear oil & $30 \%$ & 0.93 & 2.11 & 0.16 \\
\hline 11 & Wei Tang et al.(2013) & Unspecified (0.04678 Pa·s) & $5 \%$ & 0.1 & 1.02 & 0.59 \\
\hline 12 & Daniel Braun et al. (2014) & $\mathrm{PAO}$ & $10 \%$ & 0.1 & 1.04 & 0.25 \\
\hline 13 & Beomkeun Kim et al. (2014) & Mineral oil CAS8042-47-5 & $30 \%$ & 0.3 & 1.36 & 0.4 \\
\hline 14 & Manabu Wakuda et al (2003) & 5W30SJ engine oil & $15 \%$ & 0.06 & 1.04 & 0.77 \\
\hline 15 & V. Ezhilmaran et al. (2018) & 20W-50 synthetic oil & $16 \%$ & 0.31 & 1.20 & 0.5 \\
\hline 16 & Jung Won Byun et al.(2010) & oil & $5 \%$ & 0.02 & 1.00 & 0.44 \\
\hline 17 & Shuangqing Qian et al. (2010) & diesel oil & $0.1 \%$ & 0.04 & 1.00 & 0.32 \\
\hline 18 & Miki Nakano et al. (2007) & VG68 & $12.6 \%$ & 0.13 & 1.07 & 0.28 \\
\hline 19 & S. Schreck et al. (2005) & water & $50 \%$ & 0.11 & 1.22 & 0.64 \\
\hline 20 & Hengzhong Fan et al. (2014) & water & $34 \%$ & 0.30 & 1.41 & 0.84 \\
\hline 21 & Etsion,I.et al. (2004) & water & $60 \%$ & 0.10 & 1.08 & 0.33 \\
\hline 22 & H Yu et al. (2010) & CD $15 \mathrm{~W}$-40 engine oil & $10.4 \%$ & 0.02 & 1.01 & 0.60 \\
\hline 23 & Xiaolei Chen et al. (2016) & CD $15 \mathrm{~W}-40$ & $8.7 \%$ & 0.09 & 1.03 & 0.62 \\
\hline 24 & Wei Huang et al. (2012) & $10 \%$ Deionized water & $22.9 \%$ & 0.1 & 1.09 & 0.14 \\
\hline
\end{tabular}




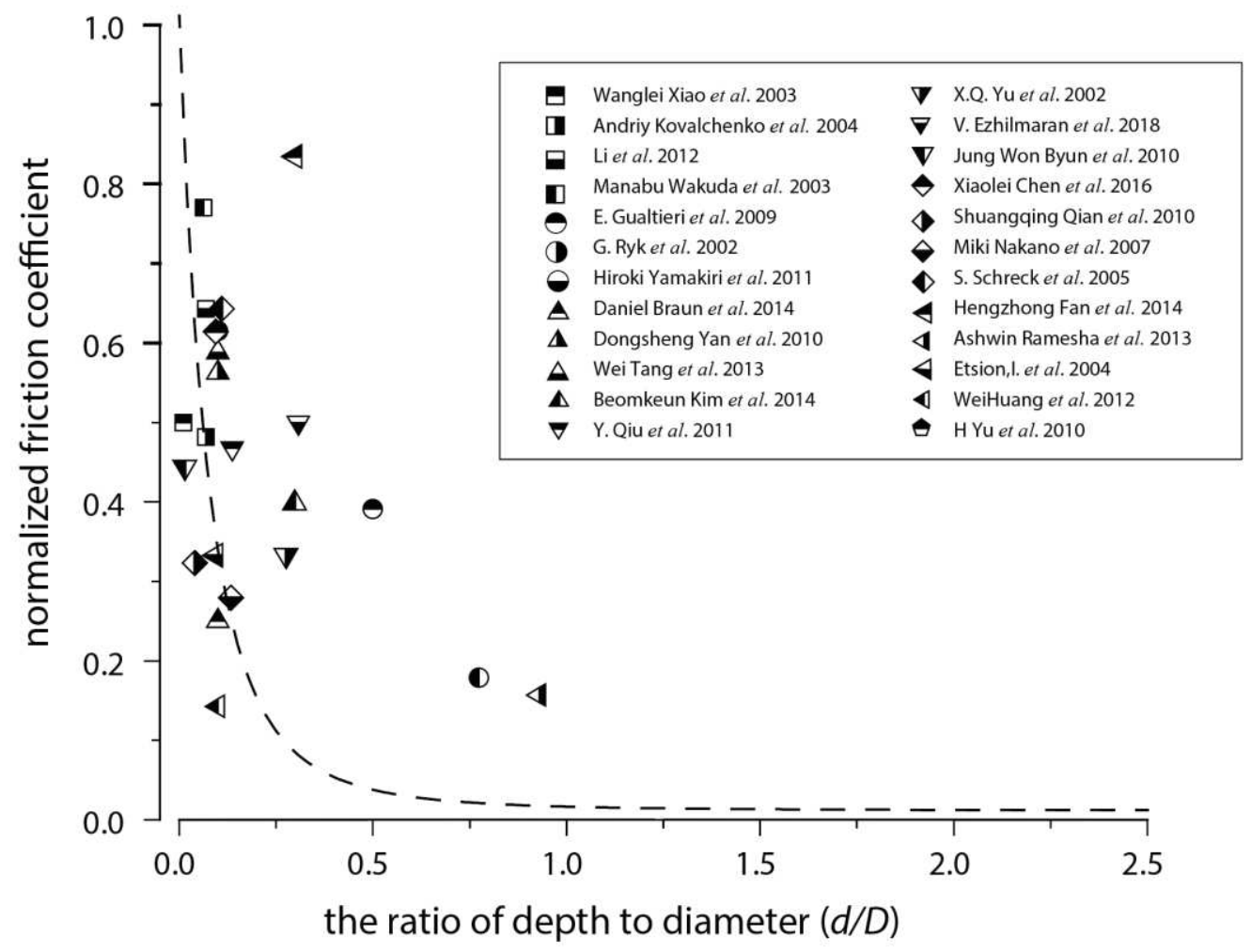

Fig. 5 Normalized friction coefficient of the best performance data within each independent study in Fig. 2 plotted against the pit depth-to-diameter ratio $(d / D)$. The same legend as in Fig. 2 was used. The lower bound of friction reduction predicted by Eq. 7 was shown as the dashed line.

\section{Acknowledgement}

The authors gratefully acknowledge financial support from the National Natural Science Foundation of China (51875153, 51975174 and 51875152) and the Fundamental Research Funds for the Central Universities (JZ2020HGTB0054).

\section{Reference}

[1] Etsion, I., Kligerman, Y., Halperin, G. Analytical and Experimental Investigation of Laser-Textured Mechanical Seal Faces. Tribology Transactions 42:511-516 (1999).

[2] Wang, X., Kato, K., Adachi, K., Aizawa, K. The effect of laser texturing of SiC surface on the critical load for the transition of water lubrication mode from hydrodynamic to mixed. Tribology International 34:703-711 (2001).

[3] Etsion, I. State of the Art in Laser Surface Texturing. Journal of Tribology 127:248-253 (2005).

[4] Wang, Q.J., Zhu, D. Virtual Texturing: Modeling the Performance of Lubricated Contacts of Engineered Surfaces. Journal of Tribology 127:722-728 (2005).

[5] Sudeep, U., Tandon, N., Pandey, R.K. Performance of Lubricated Rolling/Sliding Concentrated Contacts With Surface Textures: A Review. Journal of Tribology 137 (2015).

[6] Kovalchenko, A., Ajayi, O., Erdemir, A., Fenske, G., Etsion, I. The Effect of Laser Texturing of Steel Surfaces and Speed-Load Parameters on the Transition of Lubrication Regime from Boundary to Hydrodynamic. Tribology Transactions 47:299-307 (2004).

[7] Costa, H.L., Hutchings, I.M. Hydrodynamic lubrication of textured steel surfaces under reciprocating sliding conditions. Tribology International 40:1227-1238 (2007).

[8] Podgornik, B., Vilhena, L.M., Sedlaček, M., Rek, Z., Žun, I. Effectiveness and design of surface 
texturing for different lubrication regimes. Meccanica 47:1613-1622 (2012).

[9] Scaraggi, M., Mezzapesa, F.P., Carbone, G., Ancona, A., Tricarico, L. Friction Properties of Lubricated Laser-MicroTextured-Surfaces: An Experimental Study from Boundary- to HydrodynamicLubrication. Tribology Letters 49:117-125 (2012).

[10] Wang, W.-z., Huang, Z., Shen, D., Kong, L., Li, S. The Effect of Triangle-Shaped Surface Textures on the Performance of the Lubricated Point-Contacts. Journal of Tribology 135 (2013).

[11] Braun, D., Greiner, C., Schneider, J., Gumbsch, P. Efficiency of laser surface texturing in the reduction of friction under mixed lubrication. Tribology International 77:142-147 (2014).

[12] Scaraggi, M., Mezzapesa, F.P., Carbone, G., Ancona, A., Sorgente, D., Lugarà, P.M. Minimize friction of lubricated laser-microtextured-surfaces by tuning microholes depth. Tribology International 75:123-127 (2014).

[13] Zhang, H., Zhang, D.Y., Hua, M., Dong, G.N., Chin, K.S. A Study on the Tribological Behavior of Surface Texturing on Babbitt Alloy under Mixed or Starved Lubrication. Tribology Letters 56:305-315 (2014).

[14] Ai, X., Cheng, H.S. The Effects of Surface Texture on EHL Point Contacts. Journal of Tribology 118:59-66 (1996).

[15] Etsion, I., Burstein, L. A Model for Mechanical Seals with Regular Microsurface Structure. Tribology Transactions 39:677-683 (1996).

[16] Kligerman, Y., Etsion, I., Shinkarenko, A. Improving Tribological Performance of Piston Rings by Partial Surface Texturing. Journal of Tribology 127:632-638 (2005).

[17] de Kraker, A., van Ostayen, R.A.J., van Beek, A., Rixen, D.J. A Multiscale Method Modeling Surface Texture Effects. Journal of Tribology 129:221-230 (2007).

[18] Cupillard, S., Glavatskih, S., Cervantes, M.J. Computational fluid dynamics analysis of a journal bearing with surface texturing. Proceedings of the Institution of Mechanical Engineers, Part J: Journal of Engineering Tribology 222:97-107 (2008).

[19] Dobrica, M.B., Fillon, M. About the validity of Reynolds equation and inertia effects in textured sliders of infinite width. Proceedings of the Institution of Mechanical Engineers, Part J: Journal of Engineering Tribology 223:69-78 (2009).

[20] Han, J., Fang, L., Sun, J., Wang, Y., Ge, S., Zhu, H. Hydrodynamic Lubrication of Surfaces with Asymmetric Microdimple. Tribology Transactions 54:607-615 (2011).

[21] Papadopoulos, C.I., Kaiktsis, L., Fillon, M. Computational Fluid Dynamics Thermohydrodynamic Analysis of Three-Dimensional Sector-Pad Thrust Bearings With Rectangular Dimples. Journal of Tribology 136 (2014).

[22] Zhou, Y., Zhu, H., Zhang, W., Zuo, X., Li, Y., Yang, J. Influence of surface roughness on the friction property of textured surface. Advances in Mechanical Engineering 7 (2015).

[23] Wei, Y., Tomkowski, R., Archenti, A. Numerical Study of the Influence of Geometric Features of Dimple Texture on Hydrodynamic Pressure Generation. Metals 10 (2020).

[24] Erdemir, A. Review of engineered tribological interfaces for improved boundary lubrication. Tribology International 38:249-256 (2005).

[25] Bruzzone, A.A.G., Costa, H.L., Lonardo, P.M., Lucca, D.A. Advances in engineered surfaces for functional performance. CIRP Annals 57:750-769 (2008).

[26] Zhu, D., Jane Wang, Q. Elastohydrodynamic Lubrication: A Gateway to Interfacial MechanicsReview and Prospect. Journal of Tribology 133 (2011).

[27] Brunetière, N., Tournerie, B. Numerical analysis of a surface-textured mechanical seal operating in mixed lubrication regime. Tribology International 49:80-89 (2012).

[28] Yu, H., Huang, W., Wang, X. Dimple patterns design for different circumstances. Lubrication Science 25:67-78 (2013).

[29] Wang, L. Use of structured surfaces for friction and wear control on bearing surfaces. Surface Topography: Metrology and Properties 2 (2014).

[30] Pettersson, U., Jacobson, S. Influence of surface texture on boundary lubricated sliding contacts. Tribology International 36:857-864 (2003).

[31] Ito, S., Takahashi, K., Sasaki, S. Generation mechanism of friction anisotropy by surface texturing under boundary lubrication. Tribology International 149 (2020).

[32] Khaemba, D.N., Azam, A., See, T., Neville, A., Salehi, F.M. Understanding the role of surface textures in improving the performance of boundary additives, part I: Experimental. Tribology 
International $146(2020)$.

[33] Ramesh, A., Akram, W., Mishra, S.P., Cannon, A.H., Polycarpou, A.A., King, W.P. Friction characteristics of microtextured surfaces under mixed and hydrodynamic lubrication. Tribology International 57:170-176 (2013).

[34] Schneider, J., Braun, D., Greiner, C. Laser Textured Surfaces for Mixed Lubrication: Influence of Aspect Ratio, Textured Area and Dimple Arrangement. Lubricants 5 (2017).

[35] Joshi, G.S., Putignano, C., Gaudiuso, C., Stark, T., Kiedrowski, T., Ancona, A., et al. Effects of the micro surface texturing in lubricated non-conformal point contacts. Tribology International 127:296301 (2018).

[36] Rosenkranz, A., Grützmacher, P.G., Murzyn, K., Mathieu, C., Mücklich, F. Multi-scale surface patterning to tune friction under mixed lubricated conditions. Applied Nanoscience (2019).

[37] Mourier, L., Mazuyer, D., Lubrecht, A.A., Donnet, C., Audouard, E. Action of a femtosecond laser generated micro-cavity passing through a circular EHL contact. Wear 264:450-456 (2008).

[38] Taee, M., Torabi, A., Akbarzadeh, S., Khonsari, M.M., Badrossamay, M. On the Performance of EHL Contacts with Textured Surfaces. Tribology Letters 65 (2017).

[39] Marian, M., Grützmacher, P., Rosenkranz, A., Tremmel, S., Mücklich, F., Wartzack, S. Designing surface textures for EHL point-contacts - Transient 3D simulations, meta-modeling and experimental validation. Tribology International 137:152-163 (2019).

[40] Yu, X.Q., He, S., Cai, R.L. Frictional characteristics of mechanical seals with a laser-textured seal face. Journal of Materials Processing Technology 129:463-466 (2002).

[41] Nakano, M., Korenaga, A., Korenaga, A., Miyake, K., Murakami, T., Ando, Y., et al. Applying Micro-Texture to Cast Iron Surfaces to Reduce the Friction Coefficient Under Lubricated Conditions. Tribology Letters 28:131-137 (2007).

[42] Gualtieri, E., Borghi, A., Calabri, L., Pugno, N., Valeri, S. Increasing nanohardness and reducing friction of nitride steel by laser surface texturing. Tribology International 42:699-705 (2009).

[43] Byun, J.W., Shin, H.S., Kwon, M.H., Kim, B.H., Chu, C.N. Surface texturing by micro ECM for friction reduction. International Journal of Precision Engineering and Manufacturing 11:747-753 (2010). [44] Yan, D., Qu, N., Li, H., Wang, X. Significance of Dimple Parameters on the Friction of Sliding Surfaces Investigated by Orthogonal Experiments. Tribology Transactions 53:703-712 (2010).

[45] Qiu, Y., Khonsari, M.M. Experimental investigation of tribological performance of laser textured stainless steel rings. Tribology International 44:635-644 (2011).

[46] Yamakiri, H., Sasaki, S., Kurita, T., Kasashima, N. Effects of laser surface texturing on friction behavior of silicon nitride under lubrication with water. Tribology International 44:579-584 (2011).

[47] Li, Y. Frictional Properties of Textured Surfaces under Plane Contact. Journal of Mechanical Engineering 48 (2012).

[48] Mitchell, N., Eljach, C., Lodge, B., Sharp, J.L., Desjardins, J.D., Kennedy, M.S. Single and reciprocal friction testing of micropatterned surfaces for orthopedic device design. J Mech Behav Biomed Mater 7:106-115 (2012).

[49] Tang, W., Zhou, Y., Zhu, H., Yang, H. The effect of surface texturing on reducing the friction and wear of steel under lubricated sliding contact. Applied Surface Science 273:199-204 (2013).

[50] Chen, X., Qu, N., Hou, Z., Wang, X., Zhu, D. Friction Reduction of Chrome-Coated Surface with Micro-Dimple Arrays Generated by Electrochemical Micromachining. Journal of Materials Engineering and Performance 26:667-675 (2017).

[51] Ezhilmaran, V., Vasa, N.J., Vijayaraghavan, L. Investigation on generation of laser assisted dimples on piston ring surface and influence of dimple parameters on friction. Surface and Coatings Technology 335:314-326 (2018).

[52] Codrignani, A., Savio, D., Pastewka, L., Frohnapfel, B., van Ostayen, R. Optimization of surface textures in hydrodynamic lubrication through the adjoint method. Tribology International 148 (2020). [53] Lo, S.-W., Wilson, W.R.D. A Theoretical Model of Micro-Pool Lubrication in Metal Forming. Journal of Tribology 121:731-738 (1999).

[54] Ryk, G., Kligerman, Y., Etsion, I. Experimental Investigation of Laser Surface Texturing for Reciprocating Automotive Components. Tribology Transactions 45:444-449 (2002).

[55] Wang, X., Kato, K., Adachi, K., Aizawa, K. Loads carrying capacity map for the surface texture design of SiC thrust bearing sliding in water. Tribology International 36:189-197 (2003).

[56] Etsion, I., Halperin, G., Brizmer, V., Kligerman, Y. Experimental Investigation of Laser Surface 
Textured Parallel Thrust Bearings. Tribology Letters 17:295-300 (2004).

[57] Golloch, R., Merker, G.P., Kessen, U., Brinkmann, S. Functional properties of microstructured cylinder liner surfaces for internal combustion engines. Tribotest 11:307-324 (2005).

[58] Schreck, S., Zum Gahr, K.H. Laser-assisted structuring of ceramic and steel surfaces for improving tribological properties. Applied Surface Science 247:616-622 (2005).

[59] Yu, H., Deng, H., Huang, W., Wang, X. The effect of dimple shapes on friction of parallel surfaces. Proceedings of the Institution of Mechanical Engineers, Part J: Journal of Engineering Tribology 225:693-703 (2011).

[60] Kim, B., Chae, Y.H., Choi, H.S. Effects of surface texturing on the frictional behavior of cast iron surfaces. Tribology International 70:128-135 (2014).

[61] Hamilton, D.B., Walowit, J.A., Allen, C.M. A Theory of Lubrication by Microirregularities. Journal of Basic Engineering 88:177-185 (1966).

[62] Wakuda, M., Yamauchi, Y., Kanzaki, S., Yasuda, Y. Effect of surface texturing on friction reduction between ceramic and steel materials under lubricated sliding contact. Wear 254:356-363 (2003).

[63] Qian, S., Zhu, D., Qu, N., Li, H., Yan, D. Generating micro-dimples array on the hard chromecoated surface by modified through mask electrochemical micromachining. The International Journal of Advanced Manufacturing Technology 47:1121-1127 (2009).

[64] Huang, W., Jiang, L., Zhou, C., Wang, X. The lubricant retaining effect of micro-dimples on the sliding surface of PDMS. Tribology International 52:87-93 (2012).

[65] Fan, H., Hu, T., Zhang, Y., Fang, Y., Song, J., Hu, L. Tribological properties of micro-textured surfaces of ZTA ceramic nanocomposites under the combined effect of test conditions and environments. Tribology International 78:134-141 (2014).

[66] Ronen, A., Etsion, I., Kligerman, Y. Friction-Reducing Surface-Texturing in Reciprocating Automotive Components. Tribology Transactions 44:359-366 (2001).

[67] Etsion, I. Improving Tribological Performance of Mechanical Components by Laser Surface Texturing. Tribology Letters 17:733-737 (2004).

[68] Kovalchenko, A., Ajayi, O., Erdemir, A., Fenske, G., Etsion, I. The effect of laser surface texturing on transitions in lubrication regimes during unidirectional sliding contact. Tribology International 38:219-225 (2005).

[69] Wang, Z., Zhou, M., Xu, Y. The Study of Micro-grating and Micro-square-column on Frictional Resistance Reduction of 316L Stainless Steel Surface. Journal of Inorganic and Organometallic Polymers and Materials 23:803-807 (2013).

[70] Andersson, P., Koskinen, J., Varjus, S., Gerbig, Y., Haefke, H., Georgiou, S., et al. Microlubrication effect by laser-textured steel surfaces. Wear 262:369-379 (2007).

[71] Wenzel, R.N. Resistance of Solid Surfaces to Wetting by Water. Industrial \& Engineering Chemistry 28:988-994 (1936).

[72] Bico, J., Thiele, U., Quéré, D. Wetting of textured surfaces. Colloids and Surfaces A: Physicochemical and Engineering Aspects 206:41-46 (2002).

[73] Ijaola, A.O., Bamidele, E.A., Akisin, C.J., Bello, I.T., Oyatobo, A.T., Abdulkareem, A., et al. Wettability Transition for Laser Textured Surfaces: A Comprehensive Review. Surfaces and Interfaces $21(2020)$.

[74] Tasinkevych, M., Dietrich, S. Complete wetting of nanosculptured substrates. Phys Rev Lett 97:106102 (2006).

[75] Gang, O., Alvine, K.J., Fukuto, M., Pershan, P.S., Black, C.T., Ocko, B.M. Liquids on topologically nanopatterned surfaces. Phys Rev Lett 95:217801 (2005).

[76] Bonn, D., Eggers, J., Indekeu, J., Meunier, J., Rolley, E. Wetting and spreading. Reviews of Modern Physics 81:739-805 (2009). 
Wenzel model of contact angle:

$$
\text { roughness ratio: } r=\frac{A^{\prime}}{A} \quad \begin{gathered}
\theta^{*}<\theta<90^{\circ} \\
\cos \theta^{*}=r \cos \theta
\end{gathered}
$$

actual surface area: $A^{\prime}=\pi D d \frac{A}{L^{2}}+A$ area fraction of pits : $f=\frac{\pi D^{2}}{4 L^{2}}$

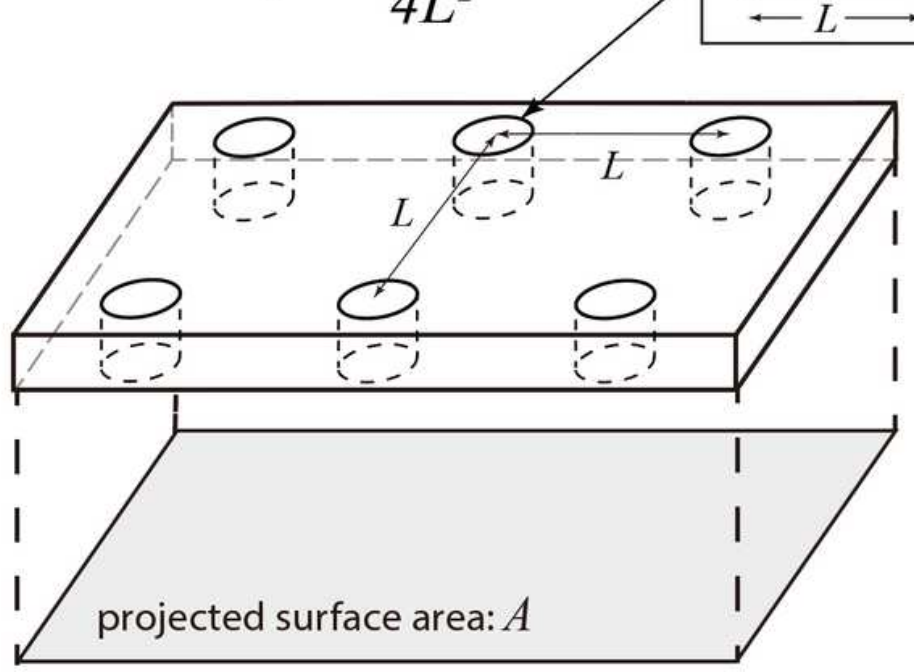

$\theta$ : Young's contact angle lubrican

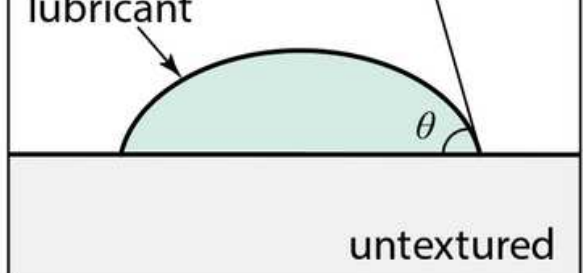

$\theta^{*}$ : effective contact angle

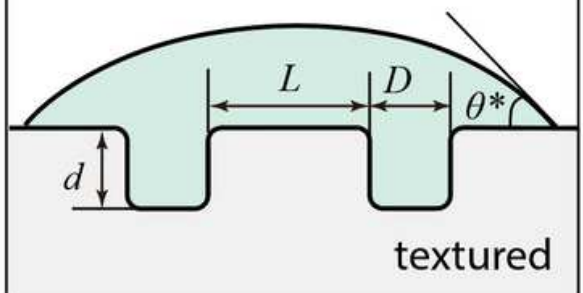

\section{Figure 1}

A simple illustration of wettability of fluid lubricants on textured surfaces based on the classical Wenzel model [71]. The roughness ratio ( $r)$ is defined as the quotient of the actual solid surface area $\left(A^{\prime}\right)$ and the projected surface area (A). Surface texture generally introduces extra area of the lubricant drop that is in contact with the solid, which causes a decrease in the wetting angle for cases where the wetting angle is less than 90 degree. 


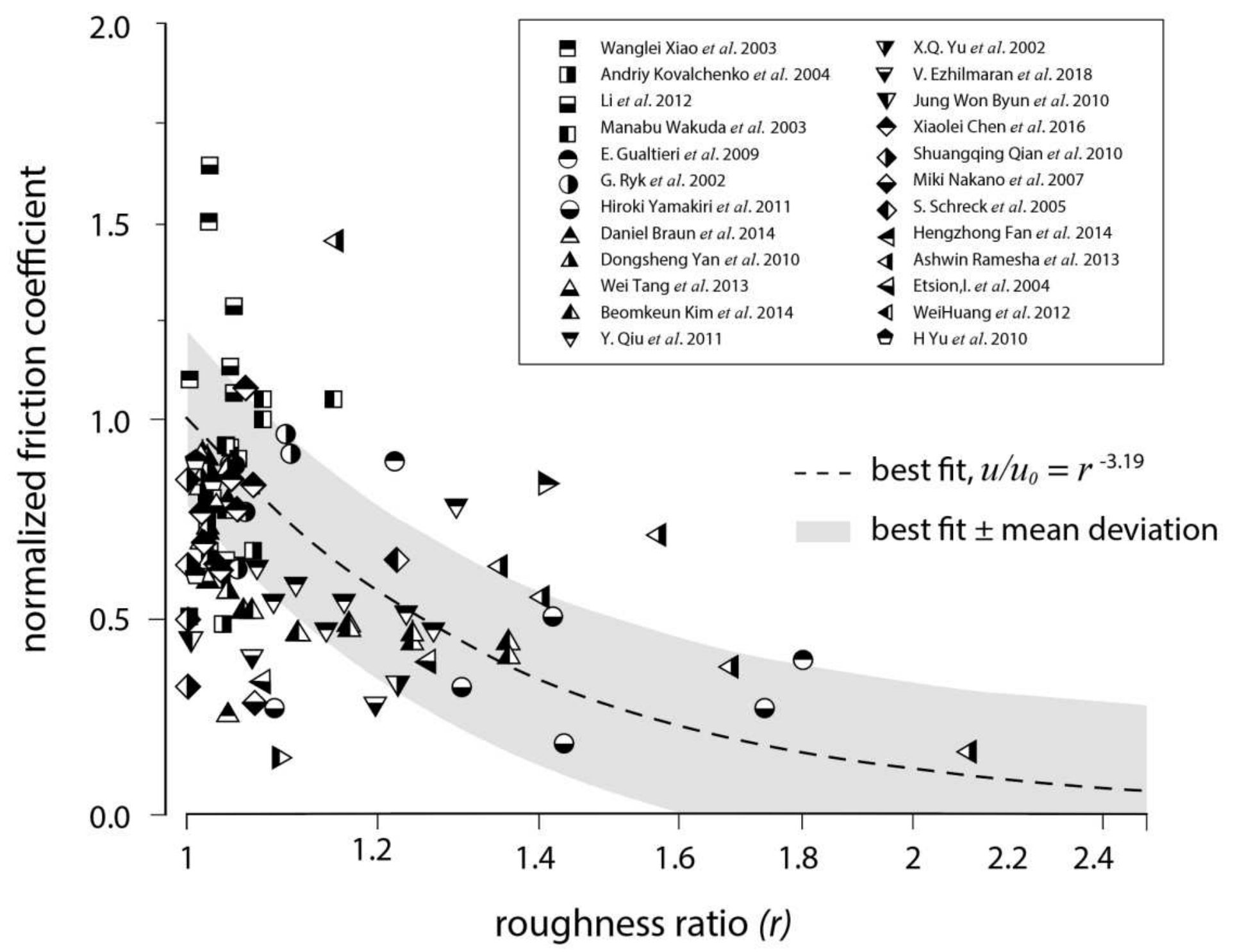

Figure 2

Normalized friction coefficient plotted against counterface roughness ratio for a collection of literature results using orthogonal arrays of micro-pits texture under fluid lubrication. Normalized friction coefficient is defined as the ratio between friction coefficient on textured counterface and that on untextured counterface under identical sliding conditions (load, speed, etc.). Counterface roughness ratio is calculated using Eq. 5 and texture parameters provided in original studies. A power law function is used to fit the data and the best fit is shown in the dashed line. Grey area represents the mean deviation of the data from the model. 


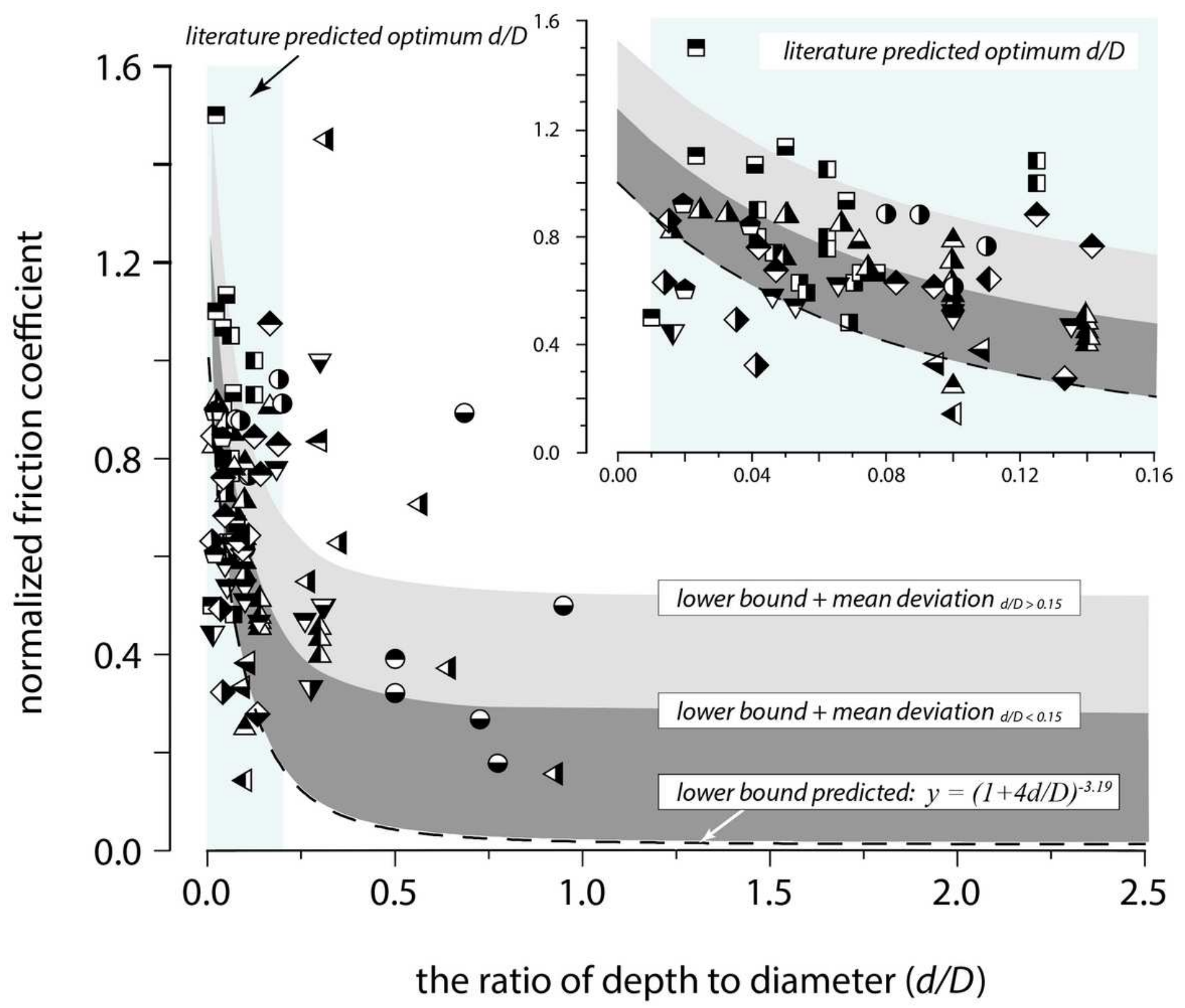

Figure 3

Normalized friction coefficient plotted against the ratio between pit depth (d) and diameter (D) for the complete dataset in Fig. 2. The same legend as in Fig. 2 was used. The lower bound of friction reduction predicted by Eq. 7 was shown as the dashed line. Grey regions represent the mean deviation of the data from the lower bound within the high ( $d / D>0.15)$ and low $(d / D<0.15)$ depth/diameter ratio domains. $91 \%$ of data points were above the predicted lower bound which supports our model of friction reduction hypothesis. 


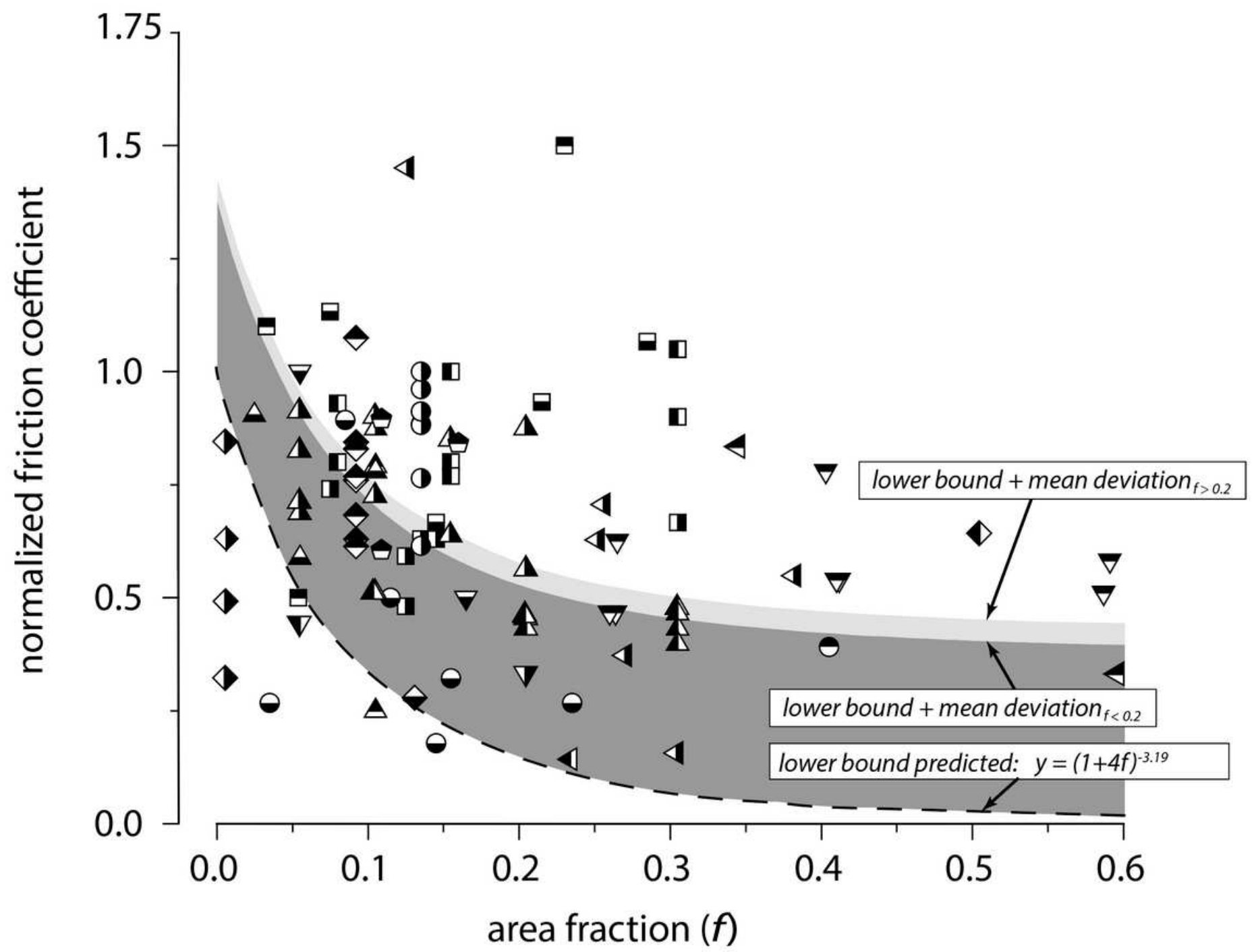

Figure 4

Normalized friction coefficient plotted against the area fraction of pits $(f)$ for the complete dataset in Fig. 2. The same legend in Fig. 2 was used. The lower bound of friction reduction predicted by Eq. 8 was shown as the dashed line. Grey regions represent the mean deviation of the data from the lower bound within the high $(f>0.2)$ and low $(f<0.2)$ pit's area fraction domains. $91 \%$ of data points were above the predicted lower bound which supports our model of friction reduction hypothesis. 


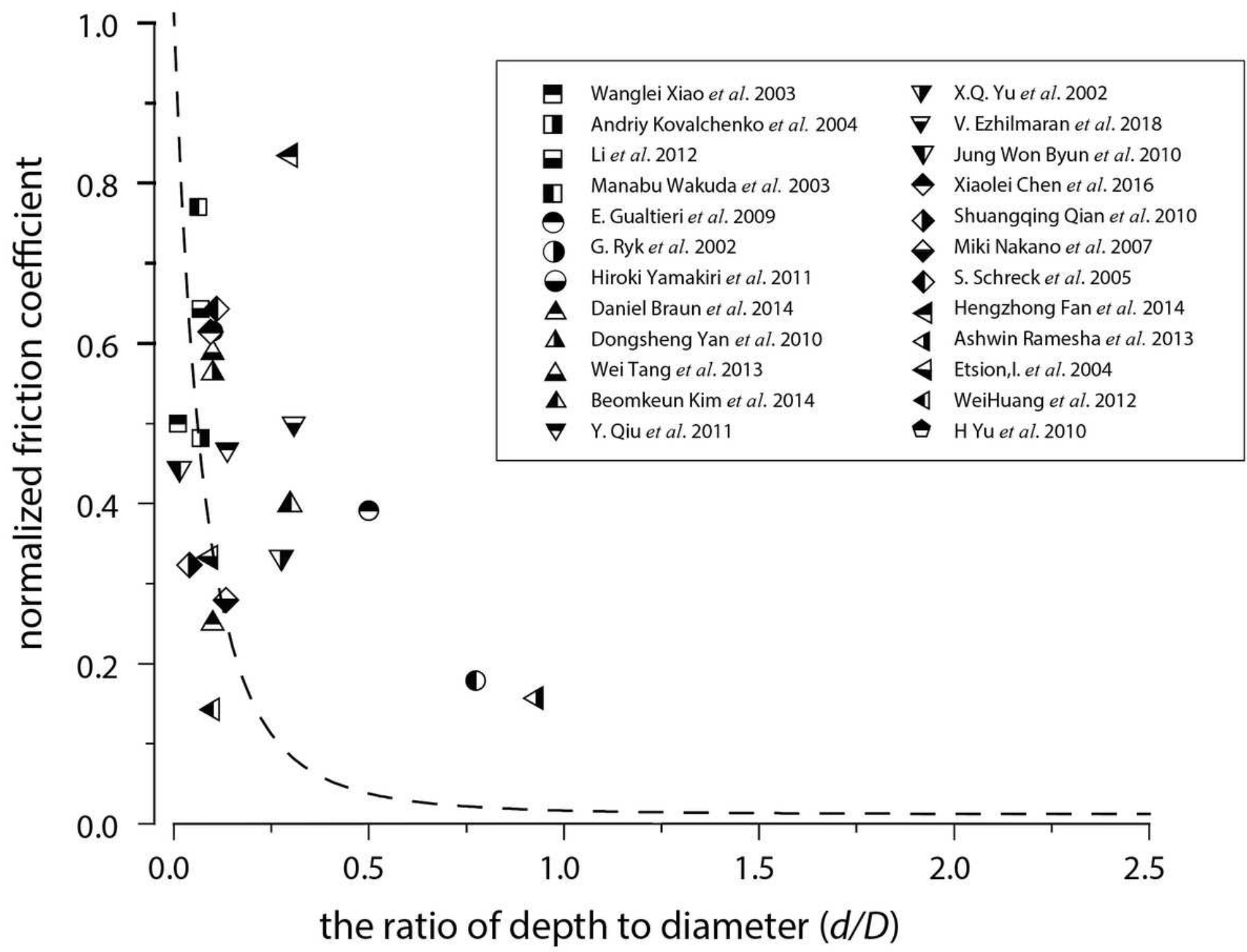

Figure 5

Normalized friction coefficient of the best performance data within each independent study in Fig. 2 plotted against the pit depth-to-diameter ratio (d/D). The same legend as in Fig. 2 was used. The lower bound of friction reduction predicted by Eq. 7 was shown as the dashed line. 